\section{Yes, uterus transplants should be publicly funded!'}

\author{
Amel Alghrani
}

Williams and Wilkinson provide an interesting paper on the latest breakthrough in the arena of assisted reproductive technologies, namely uterus transplants (UTx). The authors ask the important question of whether once UTx becomes safe enough to be offered as clinical treatment for those suffering from absolute uterine factor infertility (AUFI), publicly funded healthcare systems (such as the UK National Health Service (NHS)) should bear the financial cost of the procedure.

Rather than arguing the case for why UTx should be publicly funded, the authors instead focus on arguments on why the state should not fund UTx. They address three arguments against publicly funding UTx: (1) UTx should not be publicly funded because doing so would be inconsistent with the governments' obligations to prevent climate change and environmental pollution; (2) UTx should not be funded as it does not treat a disorder and is not medically necessary and (3) public funding for UTx should be denied because of the availability of cheaper alternatives to parenthood, such as adoption and surrogacy. They argue all three are tenuous and conclude that the case for ruling out public funding for UTx is weak.

I concurred with the excellent points made by the authors, but departed at the end when they concluded that 'surrogacy law reform could go a long way towards making surrogacy a "sufficiently good" alternative and if, such reform occurred, the case for funding UTx would be significantly weakened'.

Even in the face of surrogacy law reform, I would argue that there are strong grounds to argue for the public funding of UTx to allow women to overcome AUFI. Whether one agrees with the value placed on having genetic offspring or not, the importance of this has been explicitly acknowledged in the context of in vitro fertilisation (IVF) treatment which is publicly funded (if somewhat patchily) -The Warnock Committee reporting in 1984 noted that in 'those who long for

Correspondence to Dr Amel Alghrani, University of Liverpool School of Law \& Social Justice, Director of The Health Law \& Regulation Unit, Rendall Building, Liverpool L69 7WW, UK; a.alghrani@liverpool.ac.uk children there may be... a powerful urge to perpetuate their genes through a new generation. This desire cannot be assuaged by adoption'. ${ }^{1}$ For a woman lacking a functioning uterus the only way genetic motherhood can be achieved is through surrogacy. As the authors acknowledge, surrogacy, as a path to parenthood, is far from unproblematic. Surrogacy can be an emotionally draining method of founding a family and is shrouded in legal uncertainty. Even if this uncertainty was removed by 'better regulation', it is still not a 'sufficiently good' alternative to justify not offering state funding for UTx as research demonstrates that many women attach a great importance to the experience of gestation and pregnancy. ${ }^{2-5}$ UTx holds the potential to offer this unique experience of gestation to women and Brannstrom, the clinician who led the first successful live birth following UTx in Sweden, ${ }^{6}$ cogently sums up the advantages of UTx over surrogacy:

The advantages of a model for a successful uterine transplantation compared to gestational surrogacy are obvious for the infertile couple-apart from the joy of experiencing a pregnancy, they would not be dependent on a third party during gestation and would have full control over maternal lifestyle-influences on their offspring. Furthermore, the genetic mother, instead of the surrogate, would take the physiological risks involved with any pregnancy. Issues such as maternal bonding during gestation, the definition of motherhood and the risk of economic pressure being a factor in recruitment of the surrogate carrier, would be abolished. Also, the prospected child would not have to deal with the possible conflict of having two mothers. ${ }^{7}$

UTx allows women suffering from AUFI the opportunity to experience gestation, pregnancy and childbirth akin to their fertile female counterparts who conceive 'naturally' 5 or without assistance.

This also ties into the second argument dealt with by the authors and why I believe UTx should be publicly fundedthe argument that UTx should not be publicly funded as it is not medically necessary can be rejected by reference to the fact that many treatments are allowed on the NHS, which are not life-saving. Consider kidney transplantation, which will significantly improve a patient's quality of life, and yet dialysis is a life-preserving alternative. Cornea transplants to restore the sight of people with clouded vision is also now a well-accepted therapy and it is performed only to improve a patient's quality of life, not to preserve it. Finally, fertility treatment is publicly funded and it is worth recalling how the Warnock Committee 'took the view that actions taken with the intention of overcoming infertility can, as a rule, be regarded as acceptable substitutes for natural fertilisation'. ${ }^{8}$ This was accepted by the Government (and despite the patchy provision of IVF offered between clinical commissioning groups) IVF is publicly funded. As the Warnock Committee stated:

\footnotetext{
There are many other treatments not designed to satisfy absolute needs (in the sense the patient would die without them) which are readily available on the NHS. Medicine is no longer exclusively concerned with the preservation of life but with remedying the malfunctions of the human body. On this analysis, an inability to have children is a malfunction and should be considered in exactly the same way as any other....In summary, we conclude that infertility is a condition meriting treatment. ${ }^{9}$
}

Public funding for IVF to assist women overcome infertility is available. UTx for women suffering from AUFI should be regarded no differently to other forms of fertility treatment. Just as IVF assists couples having difficulty in conceiving, UTx once sufficiently safe will assist women having difficulty gestating and thus should similarly be publicly funded. There is no justifiable reason why only the latter should be subject to the altruism/ mercy of a surrogate and refused public funding to overcome their infertility, while the former group are assisted.

Twitter Follow Amel Alghrani at @alghrani

Competing interests None declared.

Provenance and peer review Commissioned; internally peer reviewed.

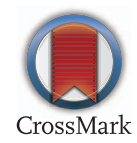

To cite Alghrani A. J Med Ethics 2016;42:566-567.

Received 29 December 2015

Accepted 6 January 2016

Published Online First 3 March 2016

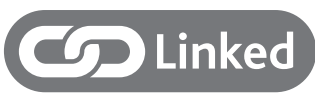

- http://dx.doi.org/10.1136/medethics-2015-102999

J Med Ethics 2016;42:566-567.

doi:10.1136/medethics-2015-103231 


\section{REFERENCES}

1 The Report of the Committee of Inquiry into Human Fertilisation and Embryology, Cm 9314 ('The Warnock Report') para 2.2. 1984.

2 Thornton JG, McNamara HM, Montague IA Would you rather be a 'birth' or a 'genetic' mother? If so, how much? J Med Ethics 1994;20:87-92.

3 Eisenberg VH, Schenker JG. Pre-embryo donation: ethical and legal aspects. Int J Gynaecol Obstet 1998;60:51-7.
4 Mahowald MB. Genes, women and equality. Oxford: Oxford University Press, 2000:129.

5 Widdows $\mathrm{H}$, MacCallum F. Disparities in parenting criteria: an exploration of the issues, focusing on adoption and embryo donation. J Med Ethics 2002;28:139-42.

6 Brannstrom M, Johannesson L, Bokstrom $H$, et al. Live Birth after uterus transplantation. Lancet 2015;385: 607-616.
7 Brännström M, Wranning CA, Racho El-Akouri R. Transplantation of the Uterus. Mol Cell Endocrinol 2003;28(202):177-84.

8 Report of the Committee of Inquiry into Human Fertilisation and Embryology (Cmnd 9314). 1984.

9 Op cit, n1 ('The Warnock Report'). para 2.4. 